\title{
Abelhas (Hymenoptera: Apoidea) visitantes florais do sabiá (Mimosa Caesalpiniifolia Benth.) em Teixeira de Freitas, Bahia, Brasil
}

Flower-visiting bees (Hymenoptera: Apoidea) of sabiá (Mimosa Caesalpiniifolia Benth.) in

Teixeira de Freitas, Bahia, Brazil

T. L. Döhler ${ }^{1}$; W. C. Pina ${ }^{1 *}$

${ }^{1}$ Universidade do Estado da Bahia - UNEB - Campus X - Departamento de Educação - Colegiado de Ciências

Biológicas - Laboratório de Zoologia. Avenida Kaikan, SN. Bairro Kaikan. CEP:45.996-023. Teixeira de Freitas-

Bahia.

*costapina@gmail.com

(Recebido em 15 de agosto de 2016; aceito em 30 de agosto de 2017)

\begin{abstract}
Mimosa caesalpiniifolia, conhecida como sabiá, está distribuída por todo nordeste brasileiro e possui grande importância econômica, ecológica e ornamental. É considerada uma planta apícola por produzir grande quantidade de pólen e néctar e visitada, principalmente, por abelhas, que são consideras agentes polinizadores de suma relevância para diversas espécies de plantas. Este estudo teve por objetivo identificar as abelhas visitantes florais do sabiazerio localizados em um fragmento de Mata Atlântica no Extremo Sul da Bahia (Teixeira de Freitas). As coletas foram realizadas entre os meses de abril e maio de 2015, entre 06:00h e 18:00 h. totalizando 108 horas. A técnica utilizada foi coleta ativa, com auxílio de rede entomológica. Foram obtidas 232 abelhas, distribuídas em 11 espécies. As espécies mais abundantes foram Trigona spinipes e Apis mellifera que foram classificadas como constantes e dominantes, as demais foram consideradas acessórias e acidentais. Os horários de maiores visitações ocorreram entre $6 \mathrm{~h}$ e $10 \mathrm{~h}$. A árvore sabiá apresentou maior pico de floração entre abril/maio e demonstra potencial para ser usada como fonte de recursos alimentares (em especial pólen). Por isso, a utilização racional da árvore sabiá poderá auxiliar na conservação e manutenção da comunidade de abelhas neste bioma.
\end{abstract}

Palavras-chave: Mimosoidae, Mata Atlântica, diversidade

Mimosa caesalpiniifolia, known as sabiá, is distributed throughout the Brazilian Northeast Region and it has great economic, ecological and ornamental importance. It is considered an apicultural plant for producing large amounts of pollen and nectar and be visited, mainly, by bees, which are considered pollinating agents of great relevance for several species of plants. This study aimed to identify flowervisiting bees of sabiá species located in a fragment of Atlantic Forest in the Extreme South of Bahia (Teixeira de Freitas). The samples were accomplished between April 2015 and May 2015, between 6 a.m. and 6 p.m., totaling 108 hours. The technique used was active collection, using entomological nets. It was obtained 232 bees, distributed in 11 species. The most abundant species were Trigona spinipes and Apis mellifera, which were classified as constant and dominant, the others were considered accessory and accidental. The schedules of major visits occurred between 6 a.m. and 10 a.m. The tree of sabiá presented highest flowering between april/may and shows potential to be used as food resource (especially pollen). Therefore, rational use of the "sabiá" tree can assist in the conservation and maintenance of the community of bees in this biome.

Keywords: Mimosoideae, Atlantic Forest, Diversity

\section{INTRODUÇÃO}

Mimosa caesalpiniifolia (Leguminosae), é uma espécie arbustivo-arbórea, espinhosa, pioneira, decídua, heliófita e com ocorrência em quase todos os tipos de solo, exceto alagados $[2,3,4]$. Ocorre naturalmente na região do Nordeste do Brasil, distribuindo-se do Maranhão ao estado da Bahia, além de ter sido introduzida com êxito nas regiões do Sul e do Sudeste [2]. Vulgarmente conhecida como sabiá, possui casca com coloração semelhante a plumagem do pássaro Turdus rufiventris [1]. Sua inflorescência é do tipo panículas de espigas terminais, com 
flores pequenas, de coloração creme-esbranquiçada, hermafroditas e, suavemente perfumadas $[2,5]$. Considerada uma planta, que produz grande quantidade de pólen e néctar $[2,4]$, e visitada, principalmente, por abelhas $[2,6]$.

As abelhas são consideradas agentes polinizadores de suma relevância para diversas espécies botânicas. Os serviços ecológicos prestados por estes insetos correspondem a cerca de $50 \%$ das plantas das florestas tropicais [7].

Estima-se que existam cerca de 30 mil espécies de abelhas em todo o planeta, com diversificadas formas de forrageamento, nidificação e comportamento social [8]. Silveira et al. (2002) [9], catalogou 1.576 espécies da apifauna brasileira, sugerindo um número aproximado de três mil espécies, em cinco famílias (Andrenidae, Colletidae, Halictidae, Megachilidae e Apidae). Apidae e Halictidae são as mais diversas e abundantes. Apesar da diversidade, o número de espécies descritas representa uma quantidade ainda muito singela para os autores.

Alguns estudos sobre visitantes e polinizadores florais têm sido desenvolvidos na família botânico Leguminosae. Silva et al. (2011) [10], forneceram importantes contribuições sobre a biologia reprodutiva do maricá (Mimosa bimucronata Kuntze, 1981) na região de Botucatu e Cachoeira Paulista, em São Paulo. Gonçalves et al. (2010) [11] e Melo-Silva et al. (2014) [12] contribuíram com informações sobre a biologia reprodutivas de espécies pertencentes a subfamília Mimosoideae em Corumbá - MS e Trindade - GO, respectivamente. Já Borges (2010) [13], levantou um comparativo sobre a biologia reprodutiva de espécies lenhosas de Leguminosae na Caatinga, onde investigou a biologia reprodutiva e seus potenciais visitantes florais em três subfamílias Caesalpinioideae, Mimosoideae e Papilonoideae. Enquanto, Cruz Neto (2007) [14], em sua pesquisa, objetivou analisar os aspectos fenológicos e a eficiência dos polinizadores diurnos e noturnos de espécies do gênero Inga Miller, 1754, incrementando os conhecimentos fenológicos e colaborando com informações importantes para realização de manejos racionais das espécies e a manutenção da diversidade biológica no estado de Alagoas.

Mimosa caesalpiniifolia, é uma espécie de grande importância econômica e ecológica, sendo denominada por Ribaski et al. (2003) [5], como 'Árvore de Múltiplo uso no Brasil'. O mesmo autor destaca esta espécie como uma das principais fontes de estacas no nordeste. Sua madeira apresenta grande teor de carbono fixo o que à qualifica como uma fonte relevante para produção de carvão vegetal. Carvalho (2007) [2], menciona a utilidade da planta para usos externos, como postes, mourões, tutores, esteios e dormentes. Este autor ainda descreve seu potencial fitoterápico, sua casca é usadas em infusão para tratamento de males das vias respiratórias.

Além disso, a espécie botânica ainda possui características ornamentais, em destaque pelo arranjo entouceirado que geralmente se apresenta, podendo ser utilizada em projetos de paisagismo [5]. Ademais, pode ser classificada como planta pioneira, altamente adaptada a diversos tipos de solo e de crescimento rápido, o que a torna uma excelente opção para projetos de reflorestamentos heterogêneos destinados a recomposição de áreas degradadas, bem como seus acúleos favorecem sua utilização como cerca viva defensiva [3, 4]. Aspectos fenológicos, visitantes florais e sucesso reprodutivo de $M$. caesalpiniifolia foram estudados em Limoeiro do Norte - CE por Blochtein et al. (2008) [15].

Este trabalho tem como objetivo estudar a diversidade de abelhas visitantes florais de $M$. caesalpiniifolia, em um remanescente florestal de Mata Atlântica, em Teixeira de Freitas, extremo Sul da Bahia, visando suas contribuições para o conhecimento da fauna e flora bem como, fornecer subsídios para pesquisas futuras.

\section{MATERIAL E MÉTODOS}

O estudo foi conduzido no município de Teixeira de Freitas, no Extremo Sul da Bahia

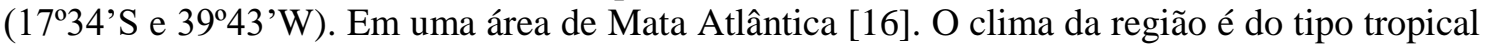
quente e úmido segundo o método de Köppen, com precipitação anual média de $1.099 \mathrm{~mm}$ com chuva bem distribuída durante o ano (com maiores médias de precipitações nos meses de novembro a janeiro) e temperaturas médias variando entre $22^{\circ} \mathrm{C}$ e $25^{\circ} \mathrm{C}$, com umidade relativa em torno de $84 \%$ [17]. 
As coletas de dados foram realizadas em um remanescente de floresta ombrófila densa, com aproximadamente 10 ha, pertencente à Universidade do Estado da Bahia - UNEB às margens da BR 101. Em suas proximidades encontram-se as instalações do Instituto Federal Baiano (IFBaiano), e também a base do Programa Arboretum de Conservação e Restauração Florestal.

Os visitantes florais de M. caesalpiniifolia, foram coletados nos meses de abril e maio de 2015, entre 06:00h e 18:00 h. Foram estabelecidos seis intervalos de horários para as coletas: (I1) $6 \mathrm{~h}-8 \mathrm{~h},(\mathrm{I}-2) 8 \mathrm{~h}-10 \mathrm{~h},(\mathrm{I}-3) 10 \mathrm{~h}-12 \mathrm{~h},(\mathrm{I}-4) 12 \mathrm{~h}-14 \mathrm{~h}$, (I-5) $14 \mathrm{~h}-16 \mathrm{~h},(\mathrm{I}-6) 16 \mathrm{~h}-18 \mathrm{~h}$. As coletas totalizaram 108 horas. Até vinte (no pico de floração) plantas foram observadas, com permanência de 10 minutos em cada indivíduo florido. A técnica utilizada foi a coleta ativa, que consiste na captura de todos indivíduos que pousam sobre as flores da planta com auxílio de rede entomológica. Está técnica, segundo Silveira et al. (2002) [9], é mais utilizada e recomendada para estudos de levantamento de abelhas no Brasil, além de ser a principal metodologia para fornecer dados qualitativos sobre as interações inseto-planta [18].

Os espécimes coletados foram mortos com acetato de etila e em seguida transferidos para sacos de papel devidamente identificados, com os respectivos dias e horários. As abelhas foram montadas em caixas entomológicas e depositadas no Laboratório de Zoologia da Universidade do Estado da Bahia, UNEB, Campus X. As identificações foram feitas de acordo com a chave de classificação proposta por Silveira et al. (2002) [9].

Para análises de dinâmica populacional de abelhas, foram utilizados os índices: riqueza (S), que corresponde ao número total de espécies de abelhas coletadas na comunidade. Frequência relativa $(\mathrm{F})$, que representa a participação do número total de indivíduos da espécie, em relação ao total de indivíduos coletados. A partir do índice de frequência, as abelhas foram classificadas em dominância (D) e não-dominante (ND). A dominância é expressa por espécies de abelhas com frequência relativa superior a $1 / \mathrm{S}$. E, Constância $(\mathrm{C})$, descrita através da porcentagem de ocorrência das espécies nos tratamentos, sendo: constantes (W) as abelhas presentes em mais de $50 \%$ das coletas; acessórias (Y), presentes em 25 a 50\% das coletas; acidentais (Z), presente em menos de 25\% das coletas. Essas análises estão de acordo com Costa (2005) [19].

\section{RESULTADOS E DISCUSSÃO}

A floração do sabiá iniciou-se na última semana do mês de março com cinco plantas floridas e teve seu término na primeira semana de junho com apenas três indivíduos com flores. A última quinzena de abril e a primeira quinzena de maio foi o pico de floração de indivíduos com vinte plantas observadas com flores. Sabe-se que a formação de flores de M. caesalpiniifolia é classificada como anual [2, 5]. Maia-Silva et al. (2012) [4], afirmou que a floração da planta ocorre em massa durante a maior parte da estação chuvosa. Dados similares foram constatados nesta área, que obteve maiores volumes de chuvas a partir do mês de março.

Foram coletadas 232 abelhas, distribuídas em 11 espécies e em duas famílias: Halictidae (02) e Apidae (230) (Tabela 1).

Blochtein et al. (2008) [15], estudando sabiá em Limoeiro do Norte - CE, obteve menor riqueza de espécies de abelhas $(\mathrm{S}=3)$ do que apresentado neste trabalho. Uma possível explicação para essa grande diferença é o esforço amostral, que nesta pesquisa estendeu-se por nove dias, totalizando 108 horas. Uma vez que, os autores supracitados coletaram os indivíduos em apenas três dias e durante $15 \mathrm{~min}$ em cada hora. Para Silveira et al. 2002 [9], maior esforço amostral, resulta em maior riqueza de grupos e maior abundância. Esta pesquisa mostrou também que a floração abrange meses, e é importante que as coletas sejam realizadas ao longo do período de florescimento pois a dinâmica populacional deve variar ao longo do período.

Estudos envolvendo visitantes florais em outras espécies de Mimosoideae também têm demostrado baixa riqueza de abelhas. Silva et al. 2011 [10], registrou 4 espécies em $M$. bimucronata em Botucatu e Cachoeira do Norte-SP; Borges (2010) [13], amostrou 2 espécies de abelhas em Anadenanthera Columbrina Brenan, 1955, em Serra Talhada-PE; Melo-Silva et al. (2014) [12], registrou 7 espécies em Leucaena leucocephala Lamark, 1961, no município de Trindade - GO. 
Tabela 1: Abundância, riqueza de espécies $(S)$, intervalos de horário, frequência $(F)$, constância $(C)$, dominância $(D)$ e número total $(N)$ de abelhas coletadas em flores de Mimosa caesalpiniifolia, entre abril e maio de 2015, em Teixeira de Freitas-BA. (I-1) 6h - 8h, (I-2) 8:01h-10h, (I-3) 10:01h - 12h, (I-4) 12:01h-14h, (I-5) 14:01h-16h, (I-6) 16:01h-18h.

\begin{tabular}{|c|c|c|c|c|c|c|c|c|c|c|c|}
\hline Família & Espécie (S) & I-1 & I-2 & I-3 & I-4 & I-5 & I-6 & $\mathbf{F}$ & $\mathbf{C}$ & D & $\mathbf{N}$ \\
\hline \multirow[t]{2}{*}{ Halictidae } & $\begin{array}{l}\text { Augochloropsis sp.1 } \\
\text { Cockerell, } 1897\end{array}$ & 01 & - & - & - & - & - & 0,00431 & $\mathrm{Z}$ & ND & 01 \\
\hline & $\begin{array}{l}\text { Augochloropsis sp.2 } \\
\text { Cockerell, } 1897\end{array}$ & - & - & 01 & - & - & - & 0,00431 & $\mathrm{Z}$ & ND & 01 \\
\hline \multirow[t]{9}{*}{ Apidae } & $\begin{array}{l}\text { Apis mellifera } \\
\text { Linnaeus } 1758\end{array}$ & 54 & 19 & 05 & - & 01 & - & 0,34051 & $\mathrm{~W}$ & $\mathrm{D}$ & 79 \\
\hline & $\begin{array}{c}\text { Bombus sp. } \\
\text { Latreille, } 1802\end{array}$ & - & 01 & - & - & 01 & - & 0,00862 & $\mathrm{Z}$ & ND & 02 \\
\hline & $\begin{array}{c}\text { Epicharis sp. Klug, } \\
1807\end{array}$ & - & - & 01 & - & - & - & 0,00431 & $\mathrm{Z}$ & ND & 01 \\
\hline & $\begin{array}{l}\text { Exomalopsis sp.1 } \\
\text { Spinola, } 1853\end{array}$ & 02 & 01 & - & - & - & - & 0,01293 & $\mathrm{Y}$ & ND & 03 \\
\hline & $\begin{array}{l}\text { Exomalopsis sp. } 2 \\
\text { Spinola, } 1853\end{array}$ & 02 & 03 & 01 & - & - & - & 0,02586 & $\mathrm{Y}$ & ND & 06 \\
\hline & $\begin{array}{l}\text { Exomalopsis sp. } 3 \\
\text { Spinola, } 1853\end{array}$ & 01 & - & 01 & - & - & - & 0,00862 & $\mathrm{Z}$ & ND & 02 \\
\hline & $\begin{array}{l}\text { Exomalopsis sp.4 } \\
\text { Spinola, } 1853\end{array}$ & - & - & 01 & - & - & - & 0,00431 & $\mathrm{Z}$ & ND & 01 \\
\hline & $\begin{array}{l}\text { Trigona spinipes } \\
\text { Fabricius } 1793\end{array}$ & 76 & 45 & 09 & 01 & 04 & - & 0,58189 & W & $\mathrm{D}$ & 135 \\
\hline & $\begin{array}{c}\text { Xylocopa } \\
\text { Neoxylocopa } \\
\text { Michener } 1954\end{array}$ & - & 01 & - & - & - & - & 0,00431 & $\mathrm{Z}$ & ND & 01 \\
\hline
\end{tabular}

$\begin{array}{llllllllllllll}\text { Total de Visitantes } & 136 & 70 & 19 & 01 & 06 & - & - & - & - & 232\end{array}$

* Constantes (W), Acessórias (Y), Acidentais (Z), Dominante (D) e Não-Dominante (ND).

As espécies supracitadas, incluindo M. caesalpiniifolia possuem alguns fatores similares, como flores de cores claras com recurso de fácil acesso e disponíveis em grande quantidade. Estas características geralmente atraem grande quantidade de diversos pequenos insetos incluindo abelhas eusociais. Abelhas com médio e grande porte geralmente são atraídas por flores grandes e vistosas [20].

Com a grande frequência de visitas de abelhas eussociais em espécies de Mimosoideae, a grande quantidade de recursos é rapidamente consumida. Isto porque abelhas que vivem em colônias com alta capacidade de recrutamento de novas operárias para forrageamento um equivalente ecológico de várias espécies solitárias, podendo desta forma, tornar a planta menos atrativa a espécies de abelhas solitárias [21]. 
Os visitantes de $M$. caesalpiniifolia mais abundantes foram $T$. spinipes (135 indivíduos) e A. mellifera (79 indivíduos), classificados como constantes e dominantes. Juntas, as duas espécies correspondem a 92,24\% dos indivíduos coletadas. As abelhas Exomalopsis sp.1 e Exomalopsis sp. 2 foram apontadas como acessórias e não-dominantes. As demais abelhas apresentaram baixos índices de constância e consideradas acidentais e não-dominantes (Tabela 1).

Resultados similares foram observados em outras espécies de Mimosoideae [10, 12, 13, 14 , 15]. Nestas espécies, os resultados de abundância de visitantes florais nas Mimosoideae podem ser explicados pela pouca disponibilidade de recursos florais de outras espécies de plantas nas áreas de coleta. Em Teixeira de Freitas, apenas $M$. caesalpiniifolia estava florescendo nos meses de abril e maio e, possivelmente, como única fonte de pólen no local. Segundo Alves (2010) [22], em ambientes com pouca disponibilidade de recursos alimentares, abelhas eussociais e com hábitos generalista, monopolizam todas as fontes de recursos florais possíveis

Vale salientar ainda que, a grande abundância de T. spinipes, pode ocasionar diminuição de abundância e riqueza de outras espécies de abelhas, devido a capacidade de suas operárias agressivas chegarem em grande número e competir intensamente com outras espécies pela fonte alimentar [23].

O período de maior intensidade de visitação às flores de $M$. caesalpiniifolia foi entre $6 \mathrm{~h}$ as 10h, representando $88,79 \%$ das coletas. É notável ainda, que ocorreu uma redução bastante acentuada após este período (Tabela 1).

Os mesmos padrões de forrageamento das abelhas foram observados em outros estudos [10, 11, 12 e 15]. Preferências de horários para forrageamento estão intrínsecas ao clima quente, uma vez que o aumento da radiação solar favorece o início do vôo ao campo [24]. Porém, a elevação excessiva da temperatura, passa a inibir as atividades dos visitantes florais, tendendo a reduzir nos horários mais quentes do dia [9], um fator que leva em consideração o custo-benefício na procura do recurso floral [25]. Ademais, no início da manhã geralmente também é o início da antese das flores/deiscência das antera, representando o período com maior quantidade de pólen/néctar disponpivel[20].

Além de temperatura, a maior visitação das abelhas no início da manhã é justificado pelo período com grande quantidade de recursos florais [23]. As flores de $M$. caesalpiniifolia abrem no início da manhã, disponibilizando grandes quantidades de recursos para as abelhas. Rosa et al. (2008) [26], constatou uma relação entre o pico de produção de recursos florais e o horário com maior frequência de visitantes florais em Caesalpinia ferrea.

\section{CONCLUSÃO}

O presente trabalho permitiu concluir que o número de espécies de abelhas foi considerável, quando comparado a outros estudos realizados com espécies botânicas da subfamília Mimosoideae. As abelhas visitantes florais mais abundantes, dominantes e constantes foram $T$. spinipes e A. mellifera. E, as abelhas apresentam uma maior atividade no período da manhã, na faixa horária (I-1) $6 \mathrm{~h}-8 \mathrm{~h}$ e (I-2) $8 \mathrm{~h}-10 \mathrm{~h}$.

Vale ressaltar que, a árvore sabiá apresentou maior pico de floração entre a última quinzena de abril e a primeira quinzena de maio e demonstra potencial para ser usada como fonte de recursos alimentares (em especial pólen), permitindo a manutenção de grandes colônias de abelhas, em um período em que a área de estudo apresenta poucas opções de plantas floridas.

\section{AGRADECIMENTOS}

Os autores agradecem o Programa Arboretum de Conservação ambiental pelo apoio e liberação da área de estudo. Agradecem também a Thiago Mahlmann pela ajuda na identificação das espécies de Augochloropsis e Exomalopsis. 


\section{REFERÊNCIAS BIBLIOGRÁFICAS}

1. Braga R. Plantas do nordeste, especialmente do Ceará. Fortaleza: Escola Superior de Agricultura de Mossoró; 1976. $540 \mathrm{p}$.

2. Carvalho PER. Sabiá (Mimosa Caesalpiniifolia) Taxonomia e Nomenclatura. Colombo: Embrapa Florestas (Circular Técnica, 135); 2007.

3. Lorenzi H. Árvores brasileiras: manual de identificação e cultivo de plantas arbóreas nativas do Brasil. Nova Odessa: Editora Plantarum; 1992. 351 p.

4. Maia-Silva C, Silva CI, Hrncir M, Queiroz RT, Imperatriz-Fonseca VL. Guia de plantas, visitadas por abelhas na caatinga. Fortaleza: Editora Fundação Brasil Cidadão; 2012. 99 p.

5. Ribaski J, Lima PCF, Oliveira VR, Drumond MA. Sabiá (Mimosa caesalpiniifolia) árvore de múltiplo uso no Brasil. Colombo: Embrapa Florestas (Comunicado Técnico, 104); 2003.

6. Aires ERB, Freitas BM. Caracterização palinológica de algumas amostras de mel do estado do Ceará. Fortaleza: Revista Ciência Agronômica. 2001;32(1):22-29.

7. Silva CI, Aleixo KP, Nunes-silva B, Freitas BM, Imperatriz-Fonseca VL. Guia ilustrado de abelhas polinizadoras no Brasil. São Paulo: Instituto Avançado da Universidade de São Paulo, Co-editor: Ministério do Meio Ambiente- Brasil; 2014. 51 p.

8. Michener CD. The bees of the world. Baltimore: The Johns Hopkins University Press; 2007. 953 p.

9. Silveira FA, Melo GAR, Almeida EAB. Abelhas brasileiras: sistemática e identificação. Belo Horizonte: Fernando A. Silveira; 2002. 253 p.

10. Silva LA, Guimarães E, Rossi MN, Maimoni-Rodella RCS. Biologia da reprodução de Mimosa bimucronata - uma espécie ruderal. Revista Planta Daninha. 2011 Mar;29(1)1011-1021, doi: http://dx.doi.org/10.1590/S0100-83582011000500007

11. Gonçalves CBS, Silva CB, Cândido ACS. Visitantes florais de Inga edulis (Fabaceae-Mimosoideae), na região do pantanal - Passo do Lontra. Revista Visão Acadêmica. 2010 Jun;11(1):14-22, doi: http://dx.doi.org/10.5380/acd.v11i1.21348

12. Melo-silva C de, Peres MP, Mesquita JN, Gonçalves BB, Leal IAB. Biologia reprodutiva de $L$. leucocephala (Lam.) R. de Wit (Fabaceae: Mimosoideae): sucesso de uma espécie invasora. Revista Neotropical Biology and Conservation. 2014:9(2):91-97, doi: http://dx.doi.org/10.4013/nbc.2014.92.03

13. Borges LA de AP. Biologia reprodutiva de espécies lenhosas de leguminosae na caatinga. Tese (PósGraduação) - Universidade Federal de Pernambuco: Recife; 2010.

14. Cruz Neto. Fenologia, biologia reprodutiva e eficiência dos visitantes florais de espécies simpátricas de Inga (Leguminosae-Mimosoideae) em remanescente de floresta atlântica no nordeste do Brasil. Tese (Pós-Graduação) - Universidade Federal de Pernambuco: Recife; 2007.

15. Blochtein B, Freitas SW, Ribeiro M de F, Vidal M de F, Cavalcante MC. Aspectos da biologia floral, visitantes florais e sucesso reprodutivo de Mimosa caesalpiniifolia (Beth) em Limoeiro do Norte, Ceará, Brasil; 2008. Capitulo 2, Biologia e ecologia da polinização; p. 137-146.

16. Fundação SOS Mata Atlântica e Instituto Nacional de Pesquisa Espaciais. Atlas dos remanescentes florestais da mata atlântica período 2012-2013. Relatório Técnico. [online]. Disponível em <http://mapas.sosma.org.br/site_media/download/atlas_2012-2013_relatorio_tecnico_2014.pdf.> Capturado em 01 de julho de 2016.

17. Campanili M, Schaffer WB. Mata Atlântica: manual de adequação ambiental. Brasília: Ministério do Meio Ambiente - Secretaria de Biodiversidade e Florestas (Biodiversidade, 35), 2010. 96 p.

18. Pinheiro-machado CF, Silveira A. Surveying and monitoring of pollinators in natural landscapes and in cultivated. Cap I, p. 25-38. In: Imperatriz-Fonseca VLI, Saraiva AM, Jong DD. (eds.). Bees as pollinators in Brazil: Assessing the status and suggesting best practices. Ribeirão Preto: Holos; 2006. $112 \mathrm{p}$.

19. Costa AJC. Abelhas (Hymenoptera: Apoidea) visitante das flores do urucum (bixa orella L.), em Vitória da Conquista - BA. Tese (Mestrado) - Universidade do Sudoeste da Bahia: Vitória da Conquista. 2005.

20. Rech AR, Agostini K, Oliveira PE, Machado IC. Biologia da polinização. 1 ed. Rio de Janeiro: Editora Projeto Cultural; 2014. Capitulo 7, Atrativos; p. 171-181.

21. Silveira FA, Campos MJO. A melissofauna de Corumbataí (SP) e Paraopeba (MG) e uma análise da biogeografia das abelhas do cerrado brasileiro (Hymenoptera, Apoidea). Revista Brasileira de Entomologia. 1995;39(2):371-401.

22. Alves LHS. Abelhas visitantes florais de Vernonia polyanthes Less. (Asteraceae), em Valença-RJ. Teses (Mestrado) - Universidade Federal Rural Rio de Janeiro: Rio de Janeiro; 2010. 
23. Imperatriz-Fonseca VL, Contrera FAL, Kleinert AMP. A meliponicultura e a iniciativa brasileira dos polinizadores. In: XV Congresso brasileiro de apicultura, $1{ }^{\circ}$ Congresso brasileiro de meliponicultura. Anais. Natal: Instituto de Biociências da Universidade de São Paulo; 2004. n.p.

24. Oliveira FL de, Dias VHP, Costa EM da, Figueira MA, Espínola-Sobrinho J. Influência das variações climáticas na atividade de vôo das abelhas jandairas Melipona subnitida Ducke (Meliponinae). Revista Ciência Agronômica. 2012 Jul/Sep;43(3):598-603. http://dx.doi.org/10.1590/S180666902012000300024

25. Carvalho-Zilse G, Porto EL, Silva CGN da, Pinto M de FC. Atividade de vôo de operárias de Melipona seminigra (Hymenoptera: Apidae) em um sistema agroflorestal da Amazônia. Revista Bioscience Journal. 2007 Nov;23(1):94-99.

26. Rosa JF, Oliveira JPL de, Alves E, Ortiz CDM. Aspectos da biologia floral, visitantes florais e sucesso reprodutivo de Mimosa caesalpiniifolia (Beth) em Limoeiro do Norte, Ceará, Brasil; 2008. Capítulo 2, Biologia e ecologia da polinização; p. 147-163. 